\title{
Magnetization Studied as a Function of Temperature and Magnetic Field for Ferromagnetic Transition in DMNaFe
}

\author{
E. KILIT DOGAN ${ }^{1,4}$ and H. YURTSEVEN (i] ${ }^{2,3,5}$ \\ 1.-Department of Physics, Van Yuzuncu Yil University, 65080 Van, Turkey. 2.-Physics Group, \\ Middle East Technical University, Northern Cyprus Campus, Kalkanli via Mersin 10, \\ Guzelyurt, Turkey. 3.-Department of Physics, Middle East Technical University, \\ 06531 Ankara, Turkey. 4.—e-mail: ekilit@yyu.edu.tr. 5.—e-mail: hamit@metu.edu.tr
}

\begin{abstract}
Magnetization has been calculated as a function of temperature in the ferromagnetic phase of $\left(\mathrm{CH}_{3}\right)_{2} \mathrm{NH}_{2} \mathrm{Na}_{0.5} \mathrm{Fe}_{0.5}(\mathrm{HCOO})_{3}$ denoted by $\mathrm{DMNaFe}$ as one of the metal formate framework by using molecular field theory. Calculated $M(T)$ is compared with the magnetization measured as a function of temperature $(H=10 \mathrm{Oe})$ in field-cooling and zero-field-cooling regimes from the literature, and a power-law analysis of the experimental data was performed for DMNaFe. Magnetization measured as a function of the magnetic field, as reported in the literature, has also been analyzed by the power-law formula. The magnetization indicates a weak first-order (or nearly second-order) ferromagnetic transition in $\mathrm{DMNaFe}$.
\end{abstract}

Key words: Ferromagnetic transition, magnetization, DMNaFe

\section{INTRODUCTION}

Hybrid organic-inorganic materials, in particular, metal-organic frameworks (MOFs), have been studied extensively because of their various structures which can be changed by using different organic ligands and metal ions. ${ }^{1-4}$ MOFs have potential applications as catalysts, chemical sensors, and luminescent materials, ${ }^{2,5,6}$ which are attractive for gas storage ${ }^{7-9}$ and they possess good gas sorption and luminescence properties, ${ }^{10,11}$ as previously indicated. ${ }^{12,13}$

MOFs are the multiferroic materials which exhibit two coexisting orders among the electric and magnetic (also elastic). Thus, as mainly oxides of transition metal elements, ${ }^{14}$ they are widely used for dynamic random access memories, data storage media, telecommunication systems, and electromagnetic sensors, etc. ${ }^{15,16}$ as also stated in an earlier study. ${ }^{17}$ As multiferroic materials, they undergo order-disorder transition, and also some perovskite MOFs, such as a heterometallic $\left(\mathrm{CH}_{3}\right)_{2} \mathrm{NH}_{2} \mathrm{Na}_{0.5}$ $\mathrm{Fe}_{0.5}(\mathrm{HCOO})_{3}$ or shortly DMNaFe, exhibit

(Received April 10, 2020; accepted August 1, 2020;

published online August 18, 2020) structural phase transition ${ }^{12,13}$ with the $R \overline{3}$ symmetry at $293 \mathrm{~K}$ and the triclinic symmetry at $110 \mathrm{~K}^{12}$ This compound undergoes a magnetic phase transition at $T_{\mathrm{m}}=8.5 \mathrm{~K}$ with a small hysteresis in the magnetization,$M(H)$, as measured, indicating a ferromagnetic character of the ordering. ${ }^{12}$ It exhibits a weak ferromagnetism due to a small canting of the underlying antiferromagnetic lattice. ${ }^{12}$ It has been pointed out ${ }^{12}$ that the small saturation magnetization of $\mathrm{DMNaFe}$, as in the other metal formates templated by dimethylammonium, ${ }^{17-20}$ ammonium, ${ }^{21}$ and imidazolium, ${ }^{22}$ is consistent with the spin-canted mechanism of the longrange magnetic ordering in DMNaFe. Magnetization of $\mathrm{DMNaFe}$ has been measured in a nominal magnetic field of 10 Oe under zero-field cooling (ZFC) and field-cooling (FC) conditions. ${ }^{12}$ Recently, we have calculated the magnetization of DMMn and chromium-doped DMMn, ${ }^{23}$ magnetization, magnetic susceptibility and the specific heat in heterometallics. ${ }^{24}$ In addition, we have investigated the magnetic ordering of the two mixed-valence iron (II)-iron (III) metal formate framework (MOFs) ${ }^{25}$

In this study, we perform a power-law analysis of the magnetization, $M(T)$, as a function of temperature by using the experimental data in the ZFC and $\mathrm{FC}$ regimes at $10 \mathrm{Oe},{ }^{12}$ and we deduce the values of 
the critical exponent, $\beta$, for the order parameter (magnetization) below $T_{\mathrm{c}}=8.5 \mathrm{~K}$ for DMNaFe. Our second analysis is the field dependence of the magnetization, $M(H)$, through the power-law formula by using the experimental data. ${ }^{12}$ From this analysis, we extract the values of the critical isotherm, $\delta$ (at $T=T_{\mathrm{c}}$ ), for DMNaFe. In the second part of this study, we calculate the temperaturedependence of the magnetization, $M(T)$, in the ZFC and $\mathrm{FC}$ regimes by means of molecular field theory (MFT) ${ }^{26,27}$ for DMNaFe. Calculated $M(T)$ is compared with the experimental data $^{12}$ for this compound.

In "Theory" section we introduce an outline of the theory. In "Calculations and results" section, calculations and results are given. Sections "Discussion and Conclusions" give our discussion and conclusions, respectively.

\section{THEORY}

In order to investigate the magnetic properties of MOFs which undergo order-disorder transitions, we employ here two theoretical models, namely, the Ising and Heisenberg models. On the basis of the classic Weiss theory of ferromagnetism, the Hamiltonian of the Heisenberg and Ising models under the field can be described as:

$$
H=-\frac{1}{2} \sum v_{i j} \overrightarrow{S_{I}} \overrightarrow{S_{j}}-\mathcal{H} \sum \overrightarrow{S_{I}} \text { Heisenberg }
$$

and:

$$
H=-\frac{1}{2} \sum v_{i j} \mu_{I} \mu_{j}-\mathcal{H} \sum \mu_{I} \text { Ising }
$$

respectively, where $v_{i j}$ is the exchange force between spins $I$ and $j, \overrightarrow{S_{I}}$ is the spin operator of the spin at site $I$ (Heisenberg model), $\mu_{I}$ is the $z$ component of spin $1 / 2$ which can take values of \pm 1 (Ising model) and $\mathcal{H}$ is the external field (in the units of energy), as given by Brout. ${ }^{26}$ Spins in the Heisenberg model are isotropically coupled, whereas in the Ising model the coupling is completely anisotropic as a classical model since all operators $\mu_{I}$ commute. $^{26}$

For crystalline structures, Yamada et al. ${ }^{28}$ has developed an Ising pseudospin-phonon coupled model by considering the $\mathrm{NH}_{4} \mathrm{Br}$ crystal according to the Hamiltonian:

$$
\begin{aligned}
H= & \frac{1}{2}\left(\sum_{\overrightarrow{k s}} p_{\overrightarrow{k s}} p_{\overrightarrow{k s}}^{*}+w_{\overrightarrow{k s}}^{2} q_{\overrightarrow{k s}} q_{\overrightarrow{k s}}^{*}\right)-\frac{1}{2} \sum_{i j} J_{i j} \sigma_{I} \sigma_{j} \\
& -\sum_{\overrightarrow{k s}} \sum_{I} \frac{w_{\overrightarrow{k s}}}{\sqrt{N}} g_{\overrightarrow{k s}} q_{\overrightarrow{k s}} \sigma_{I} e^{i \vec{k} . \overrightarrow{r_{i}}}
\end{aligned}
$$

where $p_{\overrightarrow{k s}}$ and $q_{\vec{k} s}$ denote the momentum and the coordinate of the phonon with the wave vector $\vec{k}$ and mode $s$, respectively, and $w_{\overrightarrow{k s}}$ is the characteristic frequency of the phonon. $\sigma_{i}$ and $\sigma_{j}$ represent the spin variables as $\mu_{i}$ and $J_{i j}$ is the interaction parameter as $v_{i j}$ in the Ising model (Eq. 1b). In Eq. 2, the first term describes the phonon energy, the second term with the $J_{i j}$ gives the interaction between the nearest-neighbor spins (separated by $\overrightarrow{r_{i j}}$ ) and the third term represents the phonon-pseudospin interaction with the coupling constant $g_{\overrightarrow{k s}}$.

Matsushita ${ }^{27}$ has extended the model of Yamada et al. $^{28}$ with the interaction of one phonon and one spin by considering the interactions between two spins and two phonons according to his Hamiltonian:

$$
\begin{aligned}
H= & \frac{1}{2} \sum_{\overrightarrow{k v}}\left[p^{*}(\vec{k} v) p(\vec{k} v)+w_{o}^{2}(\overrightarrow{k v}) Q^{*}(\overrightarrow{k v}) Q(\overrightarrow{k v})\right] \\
& -\frac{1}{2} \sum_{\vec{q}} J_{\mathrm{eff}}(\vec{q}) \sigma^{*}(\vec{q}) \sigma(\vec{q}) \\
& +\sum_{\vec{k} \vec{q} v v^{\prime}} K_{1, \mathrm{eff}}\left(\vec{k} \vec{q} v v^{\prime}\right) \sigma(\vec{q}) Q^{*}(\vec{k} v) Q\left(\vec{k}-\vec{q}, v^{\prime}\right) \\
& +\sum_{\vec{k} \vec{q} q^{\prime}} K_{2, \mathrm{eff}}\left(\vec{k} \vec{q} \overrightarrow{q^{\prime}} v v^{\prime}\right) \sigma(\vec{q}) \sigma\left(\overrightarrow{q^{\prime}}\right) Q^{*}(\overrightarrow{k v}) \\
& \times Q\left(\vec{k}-\vec{q}-\overrightarrow{q^{\prime}}, v^{\prime}\right)+H_{A}
\end{aligned}
$$

where

$$
J_{\mathrm{eff}}(\vec{q})=J(\vec{q})+\sum_{v}|g(\vec{q} v)|^{2}
$$

is the effective interaction parameter. In Eq. 3, the first term, in brackets, gives the interaction energy between the two phonons, and the second term is the interaction energy between the two nearestneighbor spins. The third term describes the interaction energy between the one pseudospin and the two phonons. Finally, the fourth term is the interaction energy due to the two pseudospins and two phonons. $H_{A}$ denotes the anharmonic Hamiltonian. In Eq. 3, $K_{1, \text { eff }}$ and $K_{2, \text { eff }}$ are the coefficients related to the lattice and force constants, which define the orientations of the ions (orientations of the $\mathrm{NH}_{4}{ }^{+}$in the case of $\mathrm{NH}_{4} \mathrm{Br}$ ).

Matsushita derived the damping constant from his Hamiltonian (Eq. 3) in terms of the scattering function, and predicted the line widths of the optic modes of $\mathrm{NH}_{4} \mathrm{Br}$. The two models, namely, pseudospin-phonon coupled ${ }^{29}$ and energy-fluctuation ${ }^{30}$ models were developed from Matsushita's Hamiltonian (Eq. 3). Those two models for the damping constant were used under the assumptions and simplifications to interpret the temperature-dependence of the line widths of the internal modes in the KDP-type materials. ${ }^{31,32}$ In our earlier studies, we have also applied those two models to the ammonium halides $\left(\mathrm{NH}_{4} \mathrm{Br} \text { and } \mathrm{NH}_{4} \mathrm{Cl}\right)^{33,34}$ and $\left(\mathrm{NH}_{4}\right)_{2} \mathrm{SO}_{4} \cdot{ }^{35}$ 
Regarding the temperature-dependence of the order parameter $(\mathrm{P})$, Matsushita ${ }^{27}$ also derived it near $T_{\mathrm{C}}$ from MFT, ${ }^{26}$ with the critical exponent $\beta=$ $\frac{1}{2}$ according to $P \propto\left(\frac{T-T_{\mathrm{C}}}{T_{\mathrm{C}}}\right)^{\beta}$ as follows:

$$
\begin{array}{ll}
{\left[3\left(1-\frac{T}{T_{\mathrm{C}}}\right)\right]^{\frac{1}{2} ;},} & 0<T_{\mathrm{C}}-T<T_{\mathrm{C}} \\
M=1-2 \exp \left(-2 T_{C} / T\right), & T \ll T_{\mathrm{C}} \\
0, & T>T_{C}
\end{array}
$$

with the critical temperature, $T_{\mathrm{c}}$, where the order parameter, $P$, is replaced by the magnetization, $\mathrm{M}$, for the ferromagnetic materials.

\section{CALCULATIONS AND RESULTS}

The temperature-dependence of the magnetization for the MOFs, in particular, the DMNaFe compound, can also be calculated from MFT according to Eq. 5, and its critical behavior can be analyzed near $T_{\mathrm{c}}$ by means of a power-law formula:

$$
M(T)=M_{0}^{\prime}\left(\frac{T-T_{\mathrm{C}}}{T_{\mathrm{C}}}\right)^{\beta}
$$

where $\beta$ is the critical exponent for the order parameter (magnetization) and $M_{0}^{\prime}$ is the amplitude. The field-dependence of the magnetization can also be analyzed by the power-law formula:

$$
M_{\mathrm{H}}=M_{\mathrm{O}}\left|\frac{H-H_{\mathrm{C}}}{H_{\mathrm{C}}}\right|^{1 / \delta}
$$

with the critical isotherm $\delta$ and the amplitude $M_{\mathrm{O}}$.

The temperature-dependence of the magnetization was calculated according to Eq. 5 close to the phase transition $\left(T_{\mathrm{c}}=8.5 \mathrm{~K}\right)$ for $\mathrm{DMNaFe}$ at $10 \mathrm{Oe}$. Our calculated $M(T)$ was then compared by fitting to the experimental magnetization measured in the $\mathrm{ZFC}$ and $\mathrm{FC}$ regimes of $\mathrm{DMNaFe}^{12}$ according to the relationship:

$$
\frac{M_{\mathrm{cal}}}{M_{\mathrm{O}}}=a+b\left(\frac{M_{\mathrm{obs}}}{M_{\mathrm{O}}}\right)+c\left(\frac{M_{\mathrm{obs}}}{M_{\mathrm{O}}}\right)^{2}
$$

with the fitted parameters $a, b$, and $c$ which were determined as given in Table I. Since the magnetization increases from the low temperatures toward $T_{\mathrm{c}}$ for the ZFC regime as observed experimentally, ${ }^{12}$

Table I. Values of the parameters with the uncertainties by fitting $\mathrm{M}_{\text {cal }} / \mathrm{M}_{\mathrm{O}}$ in the field-

\begin{tabular}{|c|c|c|c|}
\hline DMNaFe & $a$ & $\boldsymbol{b}$ & $-c$ \\
\hline $\mathrm{FC}$ & $0.18 \pm 0.02$ & $1.82 \pm 0.09$ & $1.04 \pm 0.09$ \\
\hline $\mathrm{ZFC}$ & $0.29 \pm 0.01$ & $1.37 \pm 0.03$ & $0.67 \pm 0.03$ \\
\hline
\end{tabular}
cooling (FC) (Eq. 5) and zero-field-cooling (ZFC) (Eq. 9) regimes to the observed $\operatorname{data}^{12}$ for DMNaFe according to Eq. 8 for the calculation of $M(T)$, we employed the disorder parameter, $D$, defined as:

$$
D=1-M(T)=1-\left[3\left(1-\frac{T}{T_{\mathrm{C}}}\right)\right]^{\frac{1}{2}}
$$

for DMNaFe. Figure 1 gives our calculated magnetization, $M(T)$, as a function of temperature in the ferromagnetic phase $\left(T<T_{\mathrm{c}}\right)$ for the FC and ZFC regimes in DMNaFe.

In this part of our study, we analyzed the magnetization, $M(T)$, at various temperatures close to $T_{\mathrm{c}}$ according to the power-law formula (Eq. 6) in the log-log scale:

$$
\ln M=\ln M_{0}^{\prime}+\beta \ln \in
$$

where the reduced temperature is $\epsilon=\left|T-T_{\mathrm{C}}\right| / T_{\mathrm{C}}$. From our analysis, we extracted values of the critical exponent $\beta$ and the amplitude $M_{0}^{\prime}$ for the $\mathrm{FC}$ and ZFC regimes of $\mathrm{DMNaFe}$ by using the experimental data. ${ }^{12}$ Values of $\beta$ and $M_{0}^{\prime}$ are given in Table II and $\ln M$ as a function of $\ln \in$ is plotted in Fig. 2 for both regimes (FC and ZFC) of DMNaFe.

For the analysis of $M(T)$ in the ZFC regime of $\mathrm{DMNaFe}$, as it grows from the negative values to zero at $T_{\mathrm{c}}=8.5 \mathrm{~K},{ }^{12}$ we used the positive value of the magnetization ratio of $M / M_{0}^{\prime}$ (Eq. 6) according to Eq. 9 with the critical exponent for the disorder parameter (D), defined as $\Delta=\beta-1$. By writing Eq. 6 in the logarithmic form:

$$
\ln \left(\frac{M}{M_{\mathrm{O}}^{\prime}}\right)=c_{\mathrm{O}}+\Delta \ln \in
$$

where $c_{\mathrm{o}}$ is a constant (intercept), we determined the values of $\Delta$ and $c_{\mathrm{o}}$ as given in Table II with the $M_{0}^{\prime}$ value, as plotted in Fig. 3.

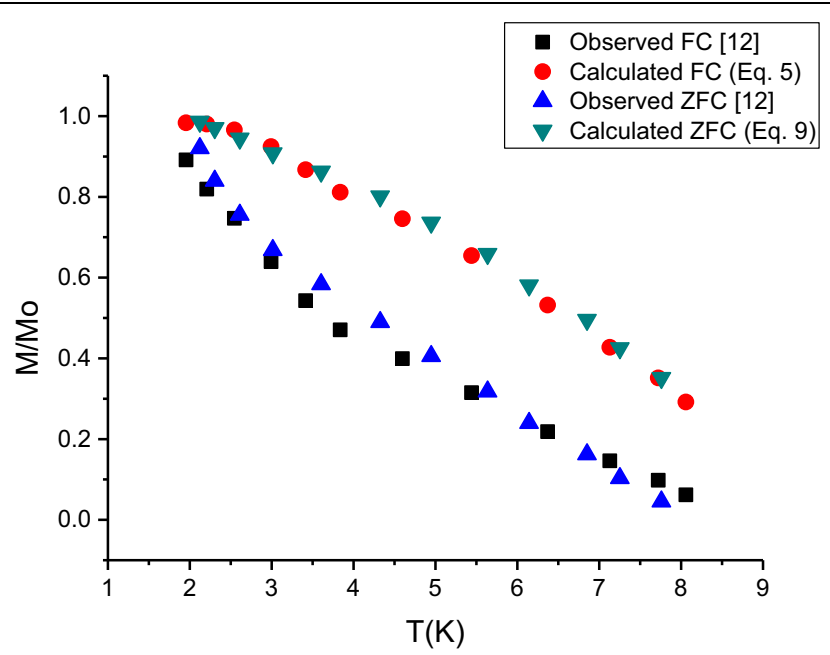

Fig. 1. Temperature variation of the magnetization $M$ (normalized) calculated by using MFT in the field-cooling $(F C)$ and zero-field cooling (ZFC) regimes of DMNaFe according to Eqs. (5) and (9), respectively. Magnetization data from Ref. 12 in the ZFC and FC regimes of this compound are also shown 
Table II. Values of the critical exponent $\beta$ for the order parameter and the amplitude $\mathrm{M}_{O}^{\prime}$ in the field-cooling (FC) regime (Eq. 10), and also values of the critical exponent $\Delta$ for the disorder parameter and the amplitude $\mathrm{M}_{\mathrm{O}}^{\prime}$ with $c_{\mathrm{o}}$ (Eq. 11) in the zero-field-cooling $(Z F C)$ regime of $\mathrm{DMNaFe}(H=10$ Oe)within the temperature interval indicated by using the experimental data ${ }^{12}$

\begin{tabular}{|c|c|c|c|c|c|c|c|}
\hline \multirow[b]{2}{*}{$\begin{array}{l}\text { Power-law analy- } \\
\text { sis }\end{array}$} & \multicolumn{3}{|r|}{ FC } & \multicolumn{4}{|c|}{ ZFC } \\
\hline & $\beta$ & $M_{\mathbf{O}}^{\prime} \underset{\mathbf{g})}{(\mathbf{e m u} /}$ & $\underset{(\mathrm{K})}{\text { Temperature interval }}$ & $\Delta$ & $c_{\mathbf{o}}$ & $\begin{array}{c}M_{\mathbf{O}}^{\prime}(\mathbf{e m u} / \\
\mathbf{g})\end{array}$ & $\underset{(\mathbf{K})}{\text { Temperature interval }}$ \\
\hline $\mathrm{DMNaFe}$ & 0.86 & 59.92 & $1.7<T<8.1$ & 0.32 & 1.27 & -116.9 & $1.9<T<7.8$ \\
\hline
\end{tabular}

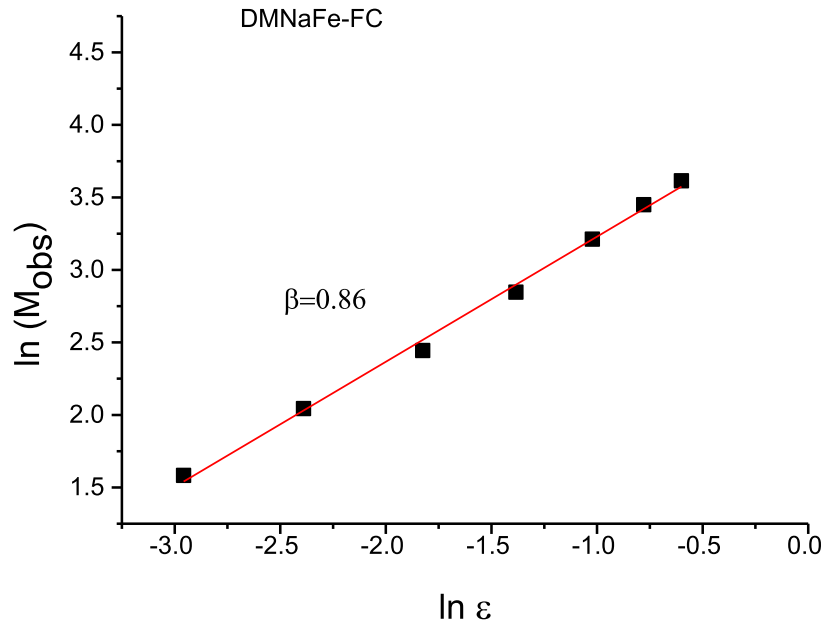

Fig. 2. Magnetization $M$ analyzed as a function of the reduced temperature $\epsilon=\left|T-T_{\mathrm{C}}\right| / T_{\mathrm{C}}$ in the log-log scale with the critical temperature $\left(T_{\mathrm{c}}=8.5 \mathrm{~K}\right)$ at $H=10$ Oe according to the power-law formula with the critical exponent $\beta$ for the order parameter (Eq. 10) by using the experimental data ${ }^{12}$ in the field-cooling $(F C)$ regime of $\mathrm{DMNaFe}$

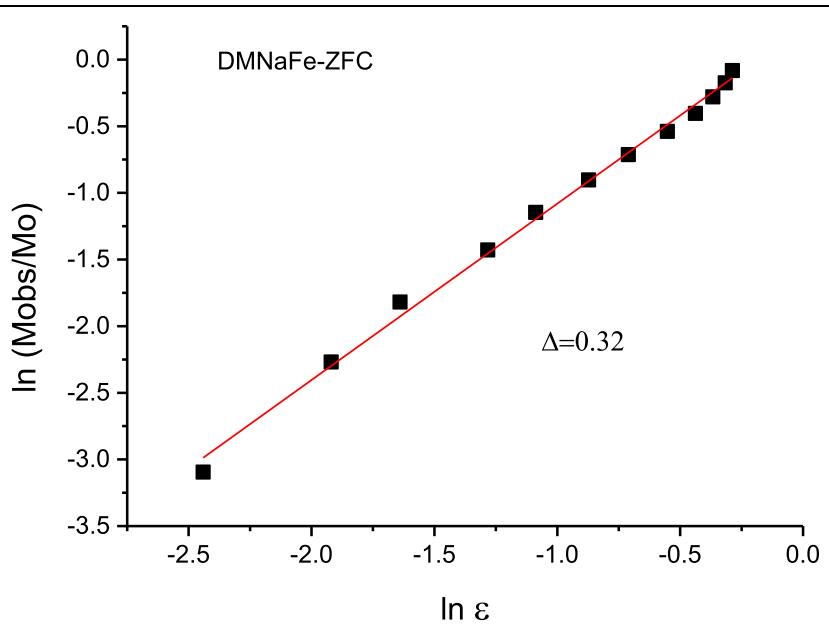

Fig. 3. Magnetization $M$ (normalized) analyzed as a function of the reduced temperature $\in=\left|T-T_{\mathrm{C}}\right| / T_{\mathrm{C}}$ in the log-log scale with the critical temperature $\left(T_{\mathrm{c}}=8.5 \mathrm{~K}\right)$ at $H=10 \mathrm{Oe}$ according to the power-law formula with the critical exponent $\Delta$ for the disorder parameter (Eq. 11) by using the experimental data ${ }^{12}$ in the zerofield-cooling $(Z F C)$ regime of $\mathrm{DMNaFe}$

We also analyzed the field-dependence of the magnetization $M(H)$ according to the power-law formula (Eq. 7) in the logarithmic form:

$$
\ln M_{\mathrm{H}}=\ln M_{\mathrm{O}}+(1 / \delta) \ln h
$$

where $h=\frac{H-H_{\mathrm{C}}}{H_{\mathrm{C}}}$ is the reduced field, and we determined using the experimental data ${ }^{12}$ the values of the critical isotherm $\delta\left(\right.$ at $\left.T_{\mathrm{c}}=8.5 \mathrm{~K}\right)$ and $M_{\mathrm{O}}$, as given in Table III. $\ln M_{\mathrm{H}}$ versus $\ln \mathrm{h}$ (Eq. 12) is plotted in Fig. 4 for DMNaFe.

\section{DISCUSSION}

Magnetization $M(T)$ calculated from MFT with the critical exponent $\beta=\frac{1}{2}$ for the order parameter (Eq. 5) describes qualitatively the critical behavior of the observed magnetization, ${ }^{12}$ which was measured as a function of temperature in the ferromagnetic phase $\left(T<T_{\mathrm{c}}\right)$ of $\mathrm{DMNaFe}$ in both the $\mathrm{FC}$ and the ZFC regimes, as shown in Fig. 1. Although quantitatively the observed and calculated $M / M_{\mathrm{O}}$ do not match, they both decrease as the transition temperature $\left(T_{\mathrm{c}}=8.5 \mathrm{~K}\right)$ is approached from the low temperatures for the $\mathrm{FC}$ and $\mathrm{ZFC}$ regimes in this compound. A quadratic fit (Eq. 8) was carried out to the observed $M(T)$ data $^{12}$ with the parameters $a, b$, and c (Table I), as shown in Fig. 1 for the FC and $\mathrm{ZFC}$ regimes of $\mathrm{DMNaFe}$. Discrepancies between the observed and calculated $M(T)$ can be due to the inadequacy of MFT with the $\beta=\frac{1}{2}$ (Eq. 5) for the magnetization (order parameter). As $M(T)$ was calculated according to Eq. (5) on the basis of the Ising pseudospin-phonon coupled model ${ }^{27}$ the Ising model is expected to give a better description of the observed data ${ }^{12}$ for the ferromagnetic transition in DMNaFe rather than the Heisenberg model, which provides an adequate description of antiferromagnetism and ferrimagnetism in insulating crystals as well as ferromagnetism in rare earths. ${ }^{26}$ In order to describe the magnetization adequately near the $T_{\mathrm{C}}$, the observed $M(T)$ data ${ }^{12}$ were analyzed according to the power-law formula with the values of $\beta$ for the order parameter in the $\mathrm{FC}$ regime (Fig. 2) and $\Delta$ for the disorder parameter in the ZFC regime (Fig. 3) with the values of $\beta$ and $\Delta$ which we determined (Table II), as stated above for DMNaFe. Our value of $\beta=0.86(\sim 0.9)$ for the $\mathrm{FC}$ regime (Fig. 2) is too large for a second-order-type of ferromagnetic-paramagnetic transition in $\mathrm{DMNaFe}$, which indicates as more likely a weakly first-order transition (nearly second-order) in this 
Table III. Values of the critical isotherm $\delta$ (at $T_{\mathrm{c}}=8.5 \mathrm{~K}$ ) and the amplitude $M_{\mathrm{o}}$ according to Eq. $12 \mathrm{within}$ the field interval indicated for DMNaFe by using the experimental data. ${ }^{12}$ The critical field $H_{c}$ is also given

\begin{tabular}{lllll}
\hline Power-law Analysis & $\frac{\delta}{1.48}$ & $\frac{\boldsymbol{M}_{\mathbf{o}}}{0.31}$ & $\frac{\boldsymbol{H}_{\mathbf{c}}(\mathbf{e m u} / \mathbf{g})}{6.49}$ & $\frac{\text { Field interval (emu/g) }}{\mathrm{DMNaFe}}$ \\
\hline
\end{tabular}

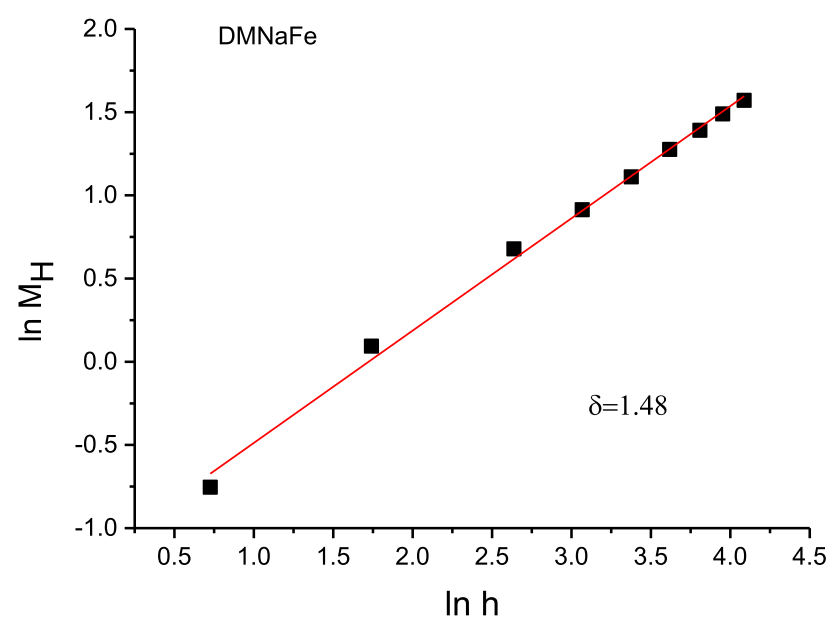

Fig. 4. Magnetization $M_{H}$ analyzed as a function of the reduced magnetic field $h=\frac{H-H_{C}}{H_{c}}$ in the log-log scale with the critical field $\left(H_{\mathrm{c}}=6.49 \mathrm{Oe}\right)$ at $T_{\mathrm{c}}^{H_{\mathrm{C}}}=8.5 \mathrm{~K}$ according to the power-law formula with the critical isotherm $\delta$ (Eq. 12) by using the experimental data ${ }^{12}$ for DMNaFe

compound. However, the value of $\Delta=0.32$ for the disorder parameter $(D)$ in the ZFC regime of $\mathrm{DMNaFe}$ is reasonable as compared to the values of $\beta(M, T)=0.326 \pm 0.004$ and $0.38 \pm 0.03$ due to the three-dimensional $(d=3)$ Ising and Heisenberg $(d=3, D=3)$ models, respectively, for a secondorder ferromagnetic transition. ${ }^{36,37}$ On the other hand, from the analysis of the magnetization $M_{H}$ as a function of the field (Eq. 7), our value of $\delta=1.48$ for the critical isotherm (at $T_{\mathrm{c}}=8.5 \mathrm{~K}$ ) of $\mathrm{DMNaFe}$ is too small in comparison with the values of $\delta(B, M)=3, \quad 4.80 \pm 0.05, \quad 4.63 \pm 0.29$ and 5 , as expected from the theoretical models of the mean field, Ising $(d=3)$, Heisenberg $(d=3, D=3)$, and spherical $(d=3, D=\infty)$, respectively. Since all these models describe ferromagnetic-paramagnetic transition of a second-order-type in the physical systems, our $M$ versus $H$ analysis also indicates a weak first-order (nearly second-order) transition according to the $M$ versus $T$ analysis given above for the ferromagnetic transition in DMNaFe. An indication of weak first-order (nearly second-order) transition in DMNaFe is also supported by the small hysteresis visible in the magnetization, which was measured as a function of the magnetic field to describe the ferromagnetic character of the ordering in this weak ferromagnet. ${ }^{12}$ For both the FC and $\mathrm{ZFC}$ regimes of $\mathrm{DMNaFe}$, the $M(H)$ curve is nonlinear in high magnetic fields. Below $T_{\mathrm{c}}$, as exhibited by some metal formate frameworks (MOFs) such as $\mathrm{DMFeCu},{ }^{13}$ short-range ordering of dimethylammonium $\left(\mathrm{DMA}^{+}\right)$cations can occur in $\mathrm{DMNaFe}$ at low temperatures, whereas, in the paramagnetic phase $\left(T>T_{\mathrm{c}}\right.$ ), the $\mathrm{DMA}^{+}$cations become dynamical disorder. Also, the large negative magnetization that occurs in the $\mathrm{ZFC}$ regime of $\mathrm{DMNaFe}$, as observed experimentally, ${ }^{12}$ is very rare in molecularbased magnets as pointed out previously, ${ }^{38}$ which was also discovered in a similar formate framework templated by $\mathrm{DMA}^{+}$cations. ${ }^{39,40}$ The negative ZFC values were considered as due to the small negative residual field of the instrument used and the easy magnetization of the sample at $H=10$ Oe. ${ }^{12} \mathrm{~A}$ possibility of the occurrence of the short-range ordering of $\mathrm{DMA}^{+}$cations and the negative magnetization in the $\mathrm{ZFC}$ regime of $\mathrm{DMNaFe}$ should be studied in some more detail in this compound.

\section{CONCLUSIONS}

The temperature-dependence of the magnetization, $M(T)$, was calculated in the ferromagnetic phase of DMNaFe by using MFT. It was compared with the magnetization measured as a function of temperature in the $\mathrm{FC}$ and the $\mathrm{ZFC}$ regimes as reported in the literature $(H=10 \mathrm{Oe})$, which we analyzed for this compound by a power-law formula. Values of the critical exponent for the order (FC) and disorder (ZFC) parameters were extracted. Analysis of the magnetization, $M(H)$, which was measured as a function of the magnetic field, was also performed by the power-law formula and the value of the critical isotherm (at $T_{\mathrm{c}}=8.5 \mathrm{~K}$ ) was extracted for the ferromagnetic transition in DMNaFe.

Our calculation and the analysis of the magnetization indicate that the ferromagnetic-paramagnetic transition in $\mathrm{DMNaFe}$ is of a weak first-order or close to a second-order. It is suggested here that calculation of the magnetization from MFT and its power-law analysis can also be performed for some other MOFs close to ferromagnetic-paramagnetic phase transitions.

\section{CONFLICT OF INTEREST}

The authors declare that they have no conflict of interest.

\section{REFERENCES}

1. P. Jain, V. Ramachandran, R.J. Clark, H.D. Zhou, B.H. Toby, N.S. Dalal, H.W. Kroto, and A.K. Cheetham, J. Am. Chem. Soc. 131, 13625 (2009).

2. L.E. Kreno, K. Leong, O.K. Farha, M. Allendorf, R.P. Van Duyne, and J.T. Hupp, Chem. Rev. 112, 1105 (2012).

3. M. Maczka, A. Ciupa, A. Gagor, A. Sieradzki, A. Pikul, B. Macalik, and M. Drozd, Inorg. Chem. 53, 5260 (2014). 
4. K. Szymborska-Malek, M. Trzebiatowska-Gutowska, M. Maczka, and A. Gagor, Spectrochimica Acta A 159, 35 (2016).

5. J.Y. Lee, O.K. Farha, J. Roberts, A.K. Scheidt, S.T. Nguyena, and J.T. Hupp, Chem. Soc. Rev. 38, 1450 (2009).

6. M.D. Allendorf, C.A. Bauer, R.K. Bhakta, and R.J.T. Houk, Chem. Soc. Rev. 38, 1330 (2009).

7. G. Rogez, N. Viart, and M. Drillon, Angew. Chem. Int. Ed. 49, 1921 (2010).

8. W. Zhang and R.G. Xiong, Chem. Rev. 112, 1163 (2012).

9. J.A. Mason, M. Veenstra, and J.R. Long, Chem. Sci. 5, 32 (2014).

10. A. Rossin, G. Giambastiani, M. Peruzzini, and R. Sessoli, Inorg. Chem. 51, 6962 (2012).

11. M. Maczka, B. Bondzior, P. Dereń, A. Sieradzki, J. Trzmiel, A. Pietraszko, and J. Hanuza, Dalton Trans. 44, 6871 (2015).

12. M. Maczka, A. Pietraszko, L. Macalik, A. Sieradzki, J. Trzmiel, and A. Pikul, Dalton Trans. 43, 17075 (2014).

13. A. Ciupa, M. Maczka, A. Gagor, A. Pikul, and M. Ptak, Dalton Trans. 44, 13234 (2015).

14. M. Maczka, M. Ptak, K. Hermanowicz, A. Majchrowski, A. Pikul, and J. Hanuza, Phys. Rev. B 83, 174439 (2011).

15. M. Fiebig, J. Phys. D 38, R123 (2005).

16. Y. Tokura, J. Magn. Magn. Mater. 310, 1145 (2007).

17. M. Maczka, A. Gagor, B. Macalik, A. Pikul, M. Ptak, and J. Hanuza, Inorg. Chem. 53, 457 (2014).

18. X.Y. Wang, L. Gan, S.W. Zhang, and S. Gao, Inorg. Chem. 43, 4615 (2004)

19. P. Jain, N.S. Dalal, B.H. Toby, H.W. Kroto, and A.K. Cheetham, J. Am. Chem. Soc. 130, 10450 (2008).

20. M. Sanchez-Andujar, S. Presedo, S. Yanez-Vilar, S. CastroGarcia, J. Shamir, and M.A. Senaris-Rodriquez, Inorg. Chem. 49, 1510 (2010).

21. G.C. Xu, W. Zhang, X.M. Ma, Y.H. Hen, L. Zhang, H.L. Cai, Z.M. Wang, R.G. Xiong, and S. Gao, J. Am. Chem. Soc. 133, 14948 (2011).

22. P. Pato-Dolan, L.C. Gomez-Aguirre, J.M. Bermudez-Garcia, M. Sanchez-Andujar, A. Fondado, J. Mira, S. Castro-Garcia, and M.A. Senaris-Rodriguez, RSC Adv. 3, 22404 (2013).
23. H. Yurtseven and E.K. Dogan, Polyhedron 154, 132 (2018).

24. E.K. Dogan and H. Yurtseven, Curr. Appl. Phys. 19, 1096 (2019).

25. H. Yurtseven and E.K. Dogan, Mater. Res. Bull. 119, 110572 (2019).

26. R. Brout, Phase Transitions (New York: Benjamin, 1965) Ch 2.

27. M. Matsushita, J. Chem. Phys. 65, 23 (1976).

28. Y. Yamada, M. Mori, and Y. Noda, J. Phys. Soc. Jap. 32, 1565 (1972).

29. G. Lahajnar, R. Blinc, and S. Zumer, Phys. Condens. Matter 18, 301 (1974).

30. G. Schaack and V. Winterfeldt, Ferroelectrics 15, 35 (1977).

31. I. Laulicht and N. Luknar, Chem. Phys. Lett. 47, 237 (1977).

32. I. Laulicht, J. Phys. Chem. Solids 39, 901 (1978).

33. H. Yurtseven, Spectrochim. Acta A 62, 910 (2005).

34. H. Yurtseven and H. Karacali, Spectrochim. Acta A 65, 421 (2006).

35. H. Yurtseven, H. Karacali, and A. Kiraci, Int. J. Mod. Phys. B 25, 2063 (2011).

36. H.E. Stanley, Introduction to Phase Transitions and Critical Phenomena (Oxford: Clarendon, 1971) Ch 3.

37. J.J. Binner, N.J. Dowrick, A.J. Fisher, and M.E.J. Newman, The Theory of Critical Phenomena: An Introduction to the Renormalization Group (New York: Oxford University Press, 1992) Chapter 3.

38. J.P. Zhao, B.W. Hu, F. Lioret, J. Tao, Q. Yang, X.F. Zhang, and X.H. Bu, Inorg. Chem. 49, 10390 (2010).

39. L. Canadillas-Delgado, O. Fabelo, J.A. Rodriguez-Velamazan, M.H. Lemee-Cailleau, S.A. Mason, E. Pardo, F. Lloret, J.P. Zhao, X.H. Bu, V. Simonet, C.V. Colin, and J. Rodriguez-Carvajal, J. Am. Chem. Soc. 134, 19772 (2012).

40. M. Maczka, A. Ciupa, A. Gagor, A. Sieradzki, A. Pikul, and M. Ptak, J. Mater. Chem. 4, 1186 (2016).

Publisher's Note Springer Nature remains neutral with regard to jurisdictional claims in published maps and institutional affiliations. 